\title{
Ratiometric analysis of in vivo retinal layer thicknesses in multiple sclerosis
}

Basanta Bhaduri

Ryan M. Nolan

Ryan L. Shelton

Lara A. Pilutti

Robert W. Motl

Stephen A. Boppart 


\title{
Ratiometric analysis of in vivo retinal layer thicknesses in multiple sclerosis
}

\author{
Basanta Bhaduri, ${ }^{a, b, c}$ Ryan M. Nolan, ${ }^{b, c}$ Ryan L. Shelton,, ${ }^{b, c}$ Lara A. Pilutti, ${ }^{d}$ Robert W. Motl, ${ }^{d, e}$ and \\ Stephen A. Boppart ${ }^{b, c, f, g, *}$ \\ andian Institute of Technology (Indian School of Mines) Dhanbad, Department of Applied Physics, Academic Complex, Dhanbad, \\ Jharkhand 826004, India \\ 'University of Illinois at Urbana-Champaign, Beckman Institute for Advanced Science and Technology, 405 North Mathews Avenue, \\ Urbana, Illinois 61801, United States \\ 'University of Illinois at Urbana-Champaign, Department of Electrical and Computer Engineering, 619 South Wright Street, Champaign, \\ Illinois 61820, United States \\ dUniversity of Illinois at Urbana-Champaign, Department of Kinesiology and Community Health, 906 South Goodwin Avenue, Urbana, \\ Illinois 61801, United States \\ eUniversity of Alabama at Birmingham, Department of Physical Therapy, 1720 2nd Avenue South, Birmingham, Alabama 35294, United States \\ fUniversity of Illinois at Urbana-Champaign, Department of Bioengineering, 1304 West Springfield Avenue, Urbana, Illinois 61801, United States \\ gUniversity of Illinois at Urbana-Champaign, Department of Internal Medicine, 506 South Mathews Avenue, Urbana, Illinois 61801, United States
}

\begin{abstract}
We performed ratiometric analysis of retinal optical coherence tomography images for the first time in multiple sclerosis (MS) patients. The ratiometric analysis identified differences in several retinal layer thickness ratios in the cohort of MS subjects without a history of optic neuritis (ON) compared to healthy control $(\mathrm{HC})$ subjects, and there was no difference in standard retinal nerve fiber layer thickness $\left(\mathrm{RNFL}_{T}\right)$. The difference in such ratios between $\mathrm{HC}$ subjects and those with mild MS-disability, without a difference in $\mathrm{RNFL}_{T}$, further suggests the possibility of using layer ratiometric analysis for detecting early retinal changes in MS. Ratiometric analysis may be useful and potentially more sensitive for detecting disease changes in MS. ๑ 2016 Society of PhotoOptical Instrumentation Engineers (SPIE) [DOI: 10.1117/1.JBO.21.9.095001]
\end{abstract}

Keywords: optical coherence tomography; multiple sclerosis; retinal layers; retinal thicknesses.

Paper 160425R received Jun. 19, 2016; accepted for publication Aug. 8, 2016; published online Sep. 2, 2016.

\section{Introduction}

Multiple sclerosis (MS) is known as an inflammatory, autoimmune, demyelinating disease of the central nervous system (CNS) and is the most common nontraumatic cause of neurological disability in early to middle adulthood. ${ }^{1}$ As the retina is part of the CNS, a broad spectrum of visual disturbances, spanning both the visual sensory and ocular motor systems, has been identified as a consequence of MS. Optic neuritis $(\mathrm{ON})$, a common initial manifestation of MS, is an immunemediated acute inflammatory disorder of the optic nerve. ${ }^{2}$ It is estimated that nearly $20 \%$ of all patients with MS present initially with $\mathrm{ON}$, and an additional $40 \%$ will have ON at some point in their disease course. ${ }^{3}$ Most patients with ON have good recovery of visual function, but demyelination, ion-channel redistribution, and axonal loss often lead to some remaining subtle signs and symptoms. Magnetic resonance imaging (MRI) provides information regarding disease burden, with emphasis on inflammation and demyelination, but its capacity to precisely quantify axonal and neuronal loss within the brain has been limited. MRI further provides essentially no information regarding disease pathology in the anterior visual pathway. ${ }^{4}$

Optical coherence tomography (OCT) $)^{5-7}$ is an established noninvasive imaging technique that can be used to identify image-based markers for diagnostics and clinical investigation of retinal diseases. Spectral domain OCT systems can capture retinal images at a faster scan rate and higher resolution to detect diseases that affect the retina, including those resulting from

*Address all correspondence to: Stephen A. Boppart, E-mail: boppart@illinois .edu axonal loss affecting the anterior optic pathway, such as MS. ${ }^{8,9}$ Since the retina is a readily accessible part of the CNS for clinical examination, the cross-sectional images of OCT are more frequently being used for retinal imaging in many eye diseases. With recent advances in algorithms for segmenting OCT images, ${ }^{10}$ quantitative measurements of retinal layer thicknesses have the potential to become useful imagebased biomarkers to assess axonal loss in patients with MS and potentially a useful method for monitoring MS progression and treatment. Several research groups have reported peripapillary retinal nerve fiber layer thickness $\left(\mathrm{RNFL}_{T}\right)$ thinning measured by $\mathrm{OCT}$ in $\mathrm{MS}^{4,11}$ and based on $\mathrm{ON},{ }^{12,13}$ that may be useful as a surrogate marker for brain atrophy. Some research supports a thinning of the ganglion cell layer (GCL) across all MS subtypes. ${ }^{9}$ Moreover, the macular inner and outer nuclear layers along with peripapillary RNFL are thinner in a subset of MS patients compared with normal controls. ${ }^{14}$

Our hypothesis is that there may be other retinal layer thicknesses demonstrating differences in MS patients when compared with healthy control (HC) subjects and patients with a history of ON. Analysis of ratios of layer thicknesses (ratiometric analysis), in particular, has recently appeared as a quantitative measure along with the thicknesses of individual layers. The GCL thickness and total retinal thickness ratio was used in diagnosing glaucoma, ${ }^{15}$ whereas neuronal remodeling of the fovea in Parkinson's disease was quantified with a measure of inner parafoveal to inner foveal thickness ratio. ${ }^{16}$ We therefore hypothesize that such ratios of different retinal layer thicknesses may 
be useful for detecting early or incremental pathology of the anterior visual pathway in MS.

Herein, we present a ratiometric analysis of retinal layers from OCT in MS and ON. We report that the $\mathrm{RNFL}_{T}$ is different in MS subjects compared to $\mathrm{HC}$ and subjects with a history of $\mathrm{ON}$. All of the thicknesses involving the RNFL are different in subjects with a history of ON when compared to MS subjects. Moreover, several ratios were different in the MS-mild disability group compared to $\mathrm{HC}$, even though there was no difference in $\mathrm{RNFL}_{T}$. We present a cohort of MS subjects that had no difference in $\mathrm{RNFL}_{T}$ when compared to $\mathrm{HC}$, but there were differences in several ratios.

\section{Methods}

\subsection{Human Subjects}

Participant recruitment occurred between February 2013 and September 2013, and MS was diagnosed based on 2010 McDonald criteria. ${ }^{17}$ We distributed flyers to participants from previous studies in our laboratory who had expressed interest in future research opportunities and to participants within the North American Research Committee on MS registry. MS patients outside of the age range of 18 to 64 years and Expanded Disability Status Scale (EDSS) ${ }^{18}$ score $>8.0$ were excluded. Participants with MS $(n=58)$ and HCs without any history of ocular or neurologic disease $(n=13)$ were recruited for the study. The analysis includes 24 eyes from 13 HC subjects, 38 eyes from 20 MS-mild patients, 31 eyes from 19 MS-moderate patients, and 36 eyes from 19 MS-severe patients. Further, 24 eyes, all in MS patients, had a history of $\mathrm{ON}$. The MS study subjects were divided into three disability groups based on the EDSS score: mild (EDSS 1.0 to 3.5), moderate (EDSS 4.0 to 5.5), and severe (EDSS 6.0 to 7.5). It is to be noted that the number of eyes is not double the number of patients or HCs. We excluded eyes that exhibited inadequate OCT signal strength on baseline scan and excluded subjects that had other ophthalmologic or neurologic disorders including glaucoma, diabetic and/or hypertensive retinopathy, and/or a refractive error greater than six diopters, from the study. Further, images with poor OCT scan centration were also excluded, as error in centration may artificially decrease or increase the measured retinal layer thicknesses. ${ }^{19}$

The protocol was reviewed and approved by the Institutional Review Board (IRB) at the University of Illinois, UrbanaChampaign, and all participants signed an informed consent

Table 1 Subject demographics and group characteristics.

\begin{tabular}{lcccc} 
& Control & Mild MS & $\begin{array}{c}\text { Moderate } \\
\text { MS }\end{array}$ & $\begin{array}{c}\text { Severe } \\
\text { MS }\end{array}$ \\
\hline Eyes (patients) & $24(13)$ & $38(20)$ & $31(19)$ & $36(19)$ \\
$\begin{array}{l}\text { Age (years) } \\
\text { Mean (SD) }\end{array}$ & $51.21(10.6)$ & $50.31(9.9)$ & $54.61(6)$ & $54.86(5.8)$ \\
ON eyes, $N(\%)$ & $0(0)$ & $4(11)$ & $13(42)$ & $7(19)$ \\
$\begin{array}{l}\text { Disease duration } \\
\text { (years) Mean (SD) }\end{array}$ & n.a. & $7.88(5.7)$ & $16.39(9.7)$ & $14.2(9.6)$ \\
$\begin{array}{l}\text { Female gender, } \\
N(\%)\end{array}$ & $22(92)$ & $23(61)$ & $24(77)$ & $28(78)$ \\
\hline
\end{tabular}

prior to participating in the study. Table 1 shows the subject demographics and group characteristics.

\subsection{Optical Coherence Tomography Imaging and Segmentation}

Retinal imaging in this study was performed using a commercial OCT system (Spectralis HRA-OCT, Heidelberg Engineering, Heidelberg, Germany). The OCT scanning protocol consisted of a 3.9-mm-diameter circumferential scan centered on the optic nerve head (total 1536 A-scans). All the OCT scans were performed by several people, all with the same level of training. After converting the images from the proprietary E2E format to tiff using the commercial viewing software (Heidelberg Eye Explorer, version 1.7.1.0), OCT images were flattened by manually fitting a line to the border between Bruch's membrane (BM) and choroid [i.e., the outer border of BM (OBBM)] in order to make all the OCT scans uniform. Figure 1 shows representative circumferential peripapillary OCT images and corresponding en face IR-SLO images, respectively, for the three study groups: (a) HC, (b) MS non-ON, and (c) MS ON subjects. The green arrows show the scan direction and were centered over the optic disc.

Six retinal layer lines: (i) inner border of internal limiting membrane (IBILM), (ii) outer (posterior) border of nerve fiber layer (OBNFL), (iii) inner border of inner plexiform layer (IBIPL), (iv) outer border of outer plexiform layer (OBOPL), (v) junction of inner and outer photoreceptor segments (ISOS), and (vi) OBBM were automatically segmented using freely available open source code [OCT Segmentation and Evaluation Graphical User Interface (OCTSEG), Pattern Recognition Lab, Friedrich-Alexander University, ErlangenNuremberg, Germany] developed based on research work on OCT. $^{20}$ We rarely corrected the segmented images manually, but did so when there was an obvious discontinuity in the automatic segmentation. Figure 2 shows representative segmented OCT images for (a) HC, (b) MS non-ON, and (c) MS ON subjects shown in Fig. 1. The six segmenting lines in Figs. 2(a)-(c), from top to bottom, represent IBILM, OBNFL, IBIPL, OBOPL, ISOS, and OBBM, respectively. The OCTSEG software generates a metadata file containing the retinal layer thicknesses in pixels, which was then read and processed in MATLAB ${ }^{\mathrm{TM}}$ to prepare the tables for analysis of variance (ANOVA).

Based on the segmentation results, 15 different retinal layer thickness combinations (IBILM-OBNFL, IBILM-IBIPL, IBILM-OBOPL, IBILM-ISOS, IBILM-OBBM, OBNFLIBIPL, OBNFL-OBOPL, OBNFL-ISOS, OBNFL-OBBM, IBIPL-OBOPL, IBIPL-ISOS, IBIPL-OBBM, OBOPL-ISOS, OBOPL-OBBM, and ISOS-OBBM) from the six retinal layer borders were possible. These thicknesses were calculated by using the global average of the thicknesses. In a similar way, there were 105 possible retinal layer thickness ratios from the 15 different thicknesses. It is noteworthy to mention that we first renamed all the OCT images with random numbers and then segmented them blindly to avoid any kind of bias. After segmentation, we regrouped the segmented images based on the key for randomization. All segmentations were performed by the same person to avoid variability.

\subsection{Statistical Analysis}

All statistical analyses were performed using SPSS (Version 22.0, IBM, Chicago, Illinois) and MATLAB ${ }^{\mathrm{TM}}$. Values are 


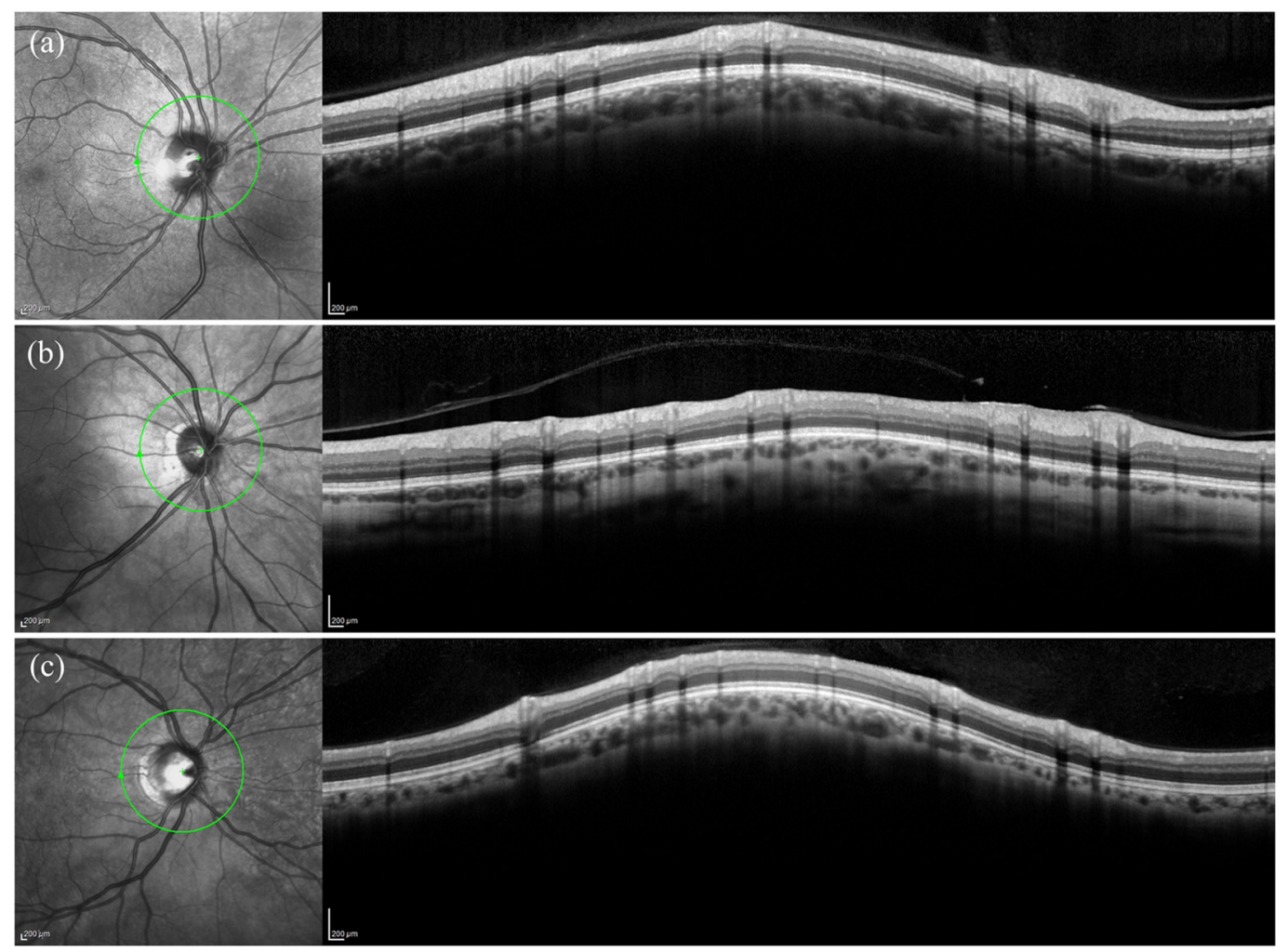

Fig. 1 Representative circumferential peripapillary OCT images and corresponding en face IR-SLO images, respectively, for (a) HC, (b) MS non-ON subjects, and (c) MS ON subjects. The green arrows show the scan direction.
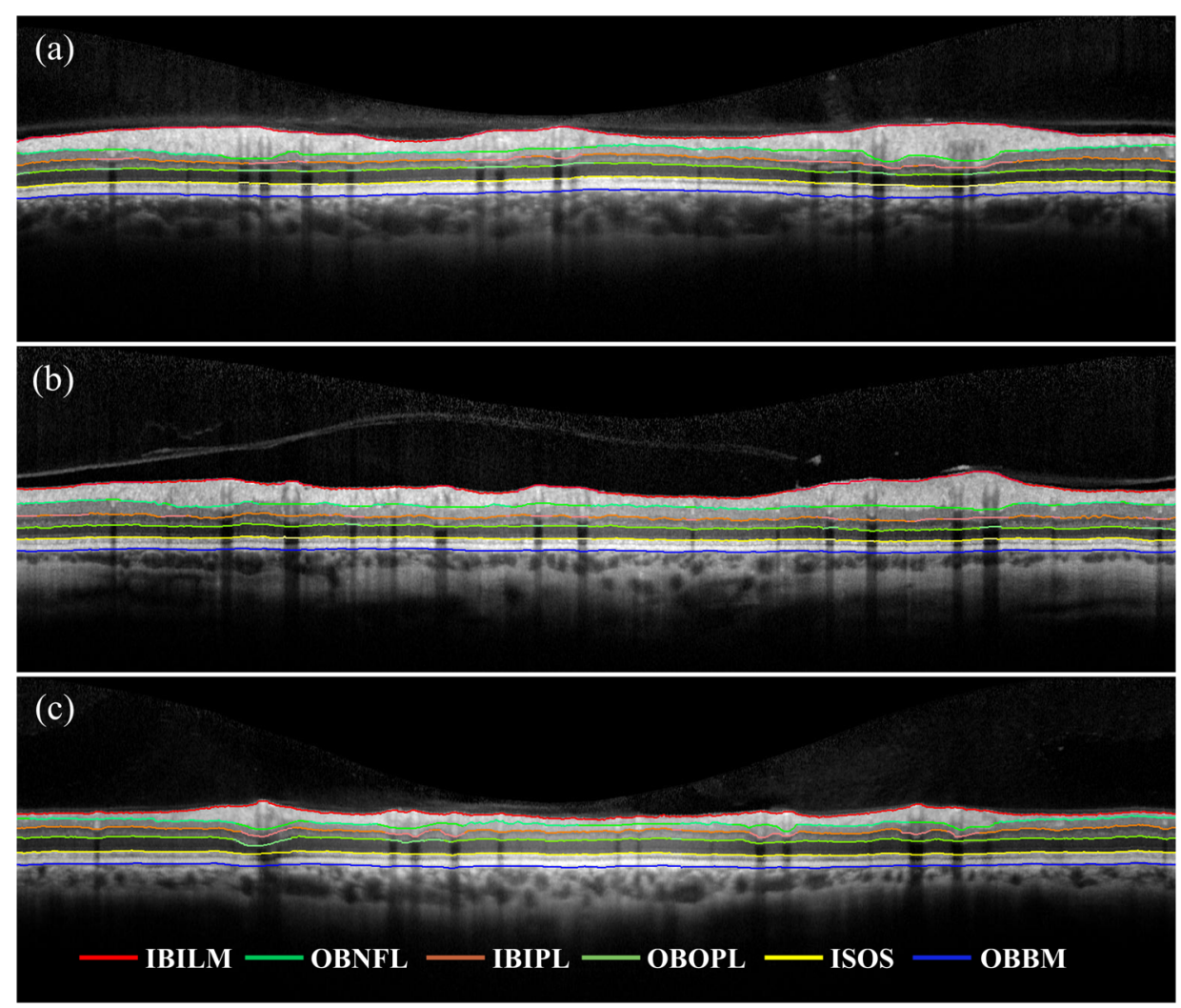

Fig. 2 Representative segmented OCT images for (a) HC, (b) MS non-ON subjects, and (c) MS ON subjects shown in Fig. 1. The six segmenting lines in (a-c), from top-to-bottom, represent IBILM, OBNFL, IBIPL, OBOPL, ISOS, and OBBM, respectively. 
presented in the text as mean (standard deviation), unless otherwise noted. A set of ANOVA was run to perform comparisons between $\mathrm{HC}$ subjects and MS non-ON subjects, and between the MS non-ON and ON groups for each of the average thicknesses and ratios. Such ANOVA was also performed to compare the MS-disability groups. Statistical significance was set at $p<0.05$. We included omega-squared $\left(\omega^{2}\right)$ as an effect size estimate for understanding the magnitude of differences between groups and interpreted the values based on the guidelines mentioned by Field. ${ }^{21}$

\section{Results}

\subsection{Comparison of Average Retinal Thicknesses}

We first compared all the retinal thicknesses (global average) among the study groups. Some of these are presented in Tables 2 and 3, which show the mean values of thicknesses along with the standard deviations in parentheses, and the $F$ and $p$ values from the one-way ANOVA as well as the $\omega^{2}$ value as effect size. Table 2 details some of the ANOVA results for the MS non-ON group with respect to $\mathrm{HC}$ subjects and for the MS ON group with respect to MS non-ON subjects. The statistically significant $\mathrm{p}$-values $(\mathrm{p}<0.05)$ are shown in bold text in Table 2 and all the other tables. It can be seen from Table 3 that the $\mathrm{RNFL}_{T}$ (i.e., the layer thickness between the IBILM and OBNFL) and the combination of $\mathrm{RNFL}_{T}$ and $\mathrm{GCL}_{T}$ (i.e., the region between IBILM and IBIPL) are different in MS non-ON subjects compared to HC. The GCL and the thicknesses involving the RNFL are different in subjects with a history of ON, compared to MS non-ON subjects. Table 3 reports such ANOVA results among the MS-disability groups: mild, moderate, and severe. From the comparison, it appears that the retinal pigment epithelium (i.e., the region between ISOS and OBBM) layer and the thicknesses involving the RNFL are different among the MS-disability groups.

\subsection{Comparison of Retinal Ratios}

Different ratios were generated from the retinal layer thicknesses (global average) and compared among the study groups. We again performed ANOVA for this with respect to $\mathrm{HC}$ for the MS non-ON group, with respect to MS non-ON for the MSON group, and also among the MS-disability groups. Since

Table 2 ANOVA results on some retinal layer thicknesses (global average) from the comparisons: MS non-ON with HCs and MS-ON with MS non-ON.

\begin{tabular}{|c|c|c|c|c|c|c|c|c|c|}
\hline \multirow[b]{3}{*}{ Thicknesses $(\mu \mathrm{m})$} & \multirow{3}{*}{$\begin{array}{c}\text { Control } \\
\text { Mean (SD) }\end{array}$} & \multicolumn{4}{|c|}{ MS non-ON } & \multicolumn{4}{|c|}{ MS-ON } \\
\hline & & \multirow[b]{2}{*}{ Mean (SD) } & \multicolumn{3}{|c|}{ ANOVA } & \multirow[b]{2}{*}{ Mean (SD) } & \multicolumn{3}{|c|}{ ANOVA } \\
\hline & & & $F$ & $p$ & $\omega^{2}$ & & $F$ & $p$ & $\omega^{2}$ \\
\hline RNFL & $101.36(5.42)$ & $93.74(11.78)$ & 9.40 & $<0.01$ & 0.07 & $80.83(19.80)$ & 15.88 & $<0.01$ & 0.12 \\
\hline IBILM-IBIPL & $144.89(6.55)$ & $137.91(14.71)$ & 5.08 & 0.03 & 0.04 & 121.63 (23.31) & 17.03 & $<0.01$ & 0.13 \\
\hline IBILM-ISOS & $256.12(10.09)$ & $252.13(18.02)$ & 1.07 & 0.30 & $<0.01$ & $236.72(25.30)$ & 11.17 & $<0.01$ & 0.09 \\
\hline IBILM-OBBM & $320.24(10.85)$ & 317.05 (19.12) & 0.60 & 0.44 & $<0.01$ & 301.33 (26.05) & 10.55 & $<0.01$ & 0.08 \\
\hline OBNFL-IBIPL & $43.53(4.87)$ & $44.17(5.48)$ & 0.26 & 0.61 & $<0.01$ & $40.80(5.98)$ & 6.72 & 0.01 & 0.05 \\
\hline IBIPL-ISOS & $111.22(6.24)$ & $114.22(7.24)$ & 3.37 & 0.07 & 0.02 & $115.09(10.17)$ & 0.22 & 0.64 & $<0.01$ \\
\hline ISOS-OBBM & $64.12(2.31)$ & $64.92(2.93)$ & 1.52 & 0.22 & $<0.01$ & $64.6(2.90)$ & 0.22 & 0.64 & $<0.01$ \\
\hline
\end{tabular}

Note: The statistically significant $p$-values $(p<0.05)$ are shown in bold text.

Table 3 ANOVA results on some retinal layer thicknesses (global average) from the comparisons: the MS-disability groups with HCs and among the disability groups.

\begin{tabular}{|c|c|c|c|c|c|c|}
\hline \multirow[b]{2}{*}{ Thicknesses $(\mu \mathrm{m})$} & \multirow{2}{*}{$\frac{\text { MS-mild }}{\text { Mean (SD) }}$} & \multirow{2}{*}{$\frac{\text { MS-moderate }}{\text { Mean (SD) }}$} & \multirow{2}{*}{$\frac{\text { MS-severe }}{\text { Mean (SD) }}$} & \multicolumn{3}{|c|}{ ANOVA } \\
\hline & & & & $F$ & $p$ & $\omega^{2}$ \\
\hline RNFL & $98.11(12.15)$ & 88.17 (10.27) & $91.92(10.53)$ & 5.27 & $<0.01$ & 0.09 \\
\hline IBILM-IBIPL & $143.53(15.37)$ & $130.37(13.25)$ & $135.81(12.33)$ & 5.83 & $<0.01$ & 0.11 \\
\hline IBILM-ISOS & 258.59 (19.95) & 243.65 (17.27) & 249.59 (13.05) & 4.97 & $<0.01$ & 0.09 \\
\hline IBILM-OBBM & $324.60(21.05)$ & $308.15(18.01)$ & 313.47 (13.69) & 5.79 & $<0.01$ & 0.10 \\
\hline OBNFL-IBIPL & $45.41(4.87)$ & $42.19(4.72)$ & $43.89(6.33)$ & 2.16 & 0.12 & 0.03 \\
\hline IBIPL-ISOS & 115.07 (7.66) & $113.28(8.96)$ & $113.78(5.47)$ & 0.4 & 0.65 & $<0.01$ \\
\hline ISOS-OBBM & $66.01(2.93)$ & 64.49 (2.69) & $63.88(2.71)$ & 4.83 & 0.01 & 0.09 \\
\hline
\end{tabular}

Note: The statistically significant $p$-values $(p<0.05)$ are shown in bold text. 
Table 4 Significant ratios involving the RFNL in both MS non-ON and MS-ON groups.

\begin{tabular}{|c|c|c|c|c|c|c|c|c|c|}
\hline \multirow[b]{2}{*}{ Ratios } & \multirow{2}{*}{$\frac{\text { Control }}{\text { Mean (SD) }}$} & \multicolumn{4}{|c|}{ MS non-ON } & \multicolumn{4}{|c|}{ MS-ON } \\
\hline & & Mean (SD) & $F$ & $p$ & $\omega^{2}$ & Mean (SD) & $F$ & $p$ & $\omega^{2}$ \\
\hline RNFL/IBILM-IBIPL & $0.68(0.03)$ & $0.65(0.03)$ & 12.15 & $<0.01$ & 0.10 & $0.63(0.05)$ & 8.7 & $<0.01$ & 0.07 \\
\hline RNFL/IBILM-ISOS & $0.39(0.02)$ & $0.36(0.03)$ & 15.27 & $<0.01$ & 0.12 & $0.33(0.05)$ & 15.41 & $<0.01$ & 0.12 \\
\hline RNFL/IBIPL-ISOS & $0.94(0.08)$ & $0.85(0.11)$ & 12.28 & $<0.01$ & 0.10 & $0.74(0.21)$ & 11.8 & $<0.01$ & 0.09 \\
\hline IBILM-OBNFL/ISOS-OBBM & $1.59(0.11)$ & $1.45(0.19)$ & 11.29 & $<0.01$ & 0.09 & $1.26(0.34)$ & 12.65 & $<0.01$ & 0.10 \\
\hline
\end{tabular}

Note: The statistically significant $p$-values $(p<0.05)$ are shown in bold text.

there were a total of 105 possible ratios, we divided them into two categories: those involving $\mathrm{RNFL}_{T}(60)$ or those that did not (45), since $\mathrm{RNFL}_{T}$ is the only single layer that is significantly different among the study groups. We subsequently identified ratios in both categories that were significantly different among the study groups.

Table 4 shows comparisons of some significant ratios involving the RFNL layer. For the MS non-ON group, ANOVA was performed with respect to $\mathrm{HC}$, whereas for MS-ON, ANOVA was performed with respect to MS non-ON. There were a total of 52 such significant ratios for both MS non-ON and MS ON groups. In contrast, Table 5 shows some significant ratios (out of a total of 23) that do not involve the RFNL layer for the MS-ON group when compared to MS non-ON. Interestingly, there were no such significant ratios for the MS non-ON group.

The ANOVA results of the $\mathrm{RNFL}_{T}$ and a few ratios among the disability groups, compared to HC subjects, are shown in
Table 6. MS-mild eyes show several significant ratios involving the RNFL layer, even though $\mathrm{RNFL}_{T}$ itself in these eyes does not change significantly with respect to $\mathrm{HC}$. The total number of such significant ratios is 14 (only three ratios are listed in Table 6). In contrast, there were 28 and 43 different ratios in MS-moderate and MS-severe groups, respectively, compared to $\mathrm{HC}$ subjects along with a different $\mathrm{RNFL}_{T}$. The ANOVA among the MS-disability groups revealed 15 different ratios (only the ratio RNFL/IBIPL-ISOS is shown in Table 6, last column and the last row) other than a different $\mathrm{RNFL}_{T}$.

Finally, Table 7 shows the ANOVA results for the cohort of MS non-ON eyes $(n=24)$ with $\mathrm{RNFL}_{T}$ (global average) in the range of 96 to $106 \mu \mathrm{m}$, when compared to $\mathrm{HC}$ subjects $(101 \pm 5 \mu \mathrm{m})$. The table shows the $\mathrm{RNFL}_{T}$ and the few significant ratios involving the RNFL layer. Statistically significant thickness ratios $(p<0.05)$ are shown in bold numbers in Table 7. It is important to mention that none of the other thicknesses are different in this cohort.

Table 5 Ratios not involving the RFNL in both MS non-ON and ON groups, but significant only for the MS-ON group.

\begin{tabular}{|c|c|c|c|c|c|c|c|c|c|}
\hline \multirow[b]{2}{*}{ Ratios } & \multirow{2}{*}{$\frac{\text { Control }}{\text { Mean (SD) }}$} & \multicolumn{4}{|c|}{ MS non-ON } & \multicolumn{4}{|c|}{ MS-ON } \\
\hline & & Mean (SD) & $F$ & $p$ & $\omega^{2}$ & Mean (SD) & $F$ & $p$ & $\omega^{2}$ \\
\hline OBNFL-IBIPL/OBNFL-OBOPL & $0.49(0.02)$ & $0.48(0.02)$ & 0.23 & 0.63 & $<0.01$ & $0.46(0.03)$ & 8.45 & $<0.01$ & 0.07 \\
\hline OBNFL-OBOPL/IBIPL-OBOPL & $1.98(0.09)$ & $1.97(0.09)$ & 0.13 & 0.71 & $<0.01$ & $1.91(0.11)$ & 6.15 & 0.01 & 0.06 \\
\hline OBNFL-ISOS/IBIPL-ISOS & $1.39(0.03)$ & $1.39(0.04)$ & 0.17 & 0.68 & $<0.01$ & $1.36(0.04)$ & 9.34 & $<0.01$ & 0.07 \\
\hline OBNFL-OBBM/IBIPL-OBBM & $1.25(0.02)$ & $1.25(0.03)$ & 0.06 & 0.81 & $<0.01$ & $1.23(0.03)$ & 8.30 & $<0.01$ & 0.07 \\
\hline
\end{tabular}

Note: The statistically significant $p$-values $(p<0.05)$ are shown in bold text.

Table 6 ANOVA results from the comparison of RNFL thickness and ratios: the MS-disability groups with HCs and among the disability groups.

\begin{tabular}{|c|c|c|c|c|c|c|c|c|c|c|c|c|c|c|c|c|}
\hline \multirow[b]{2}{*}{ Variables } & \multirow{2}{*}{$\frac{\text { Control }}{\text { Mean (SD) }}$} & \multicolumn{4}{|c|}{ MS-mild } & \multicolumn{4}{|c|}{ MS-moderate } & \multicolumn{4}{|c|}{ MS-severe } & \multicolumn{3}{|c|}{ ANOVA } \\
\hline & & Mean (SD) & $F$ & $p$ & $\omega^{2}$ & Mean (SD) & $F$ & $p$ & $\omega^{2}$ & Mean (SD) & $F$ & $p$ & $\omega^{2}$ & $F$ & $p$ & $\omega^{2}$ \\
\hline $\mathrm{RNFL}_{T}(\mu \mathrm{m})$ & $101.36(5.4)$ & $98.11(12.1)$ & 1.5 & 0.23 & 0.01 & $88.17(10.3)$ & 28.9 & $<0.01$ & 0.40 & $91.92(10.5)$ & 15.8 & $<0.01$ & 0.22 & 5.27 & $<0.01$ & 0.09 \\
\hline RNFL/IBILM-IBIPL & $0.68(0.03)$ & $0.66(0.03)$ & 7.89 & 0.01 & 0.10 & $0.65(0.03)$ & 12.8 & $<0.01$ & 0.22 & $0.65(0.04)$ & 7.73 & 0.01 & 0.11 & 0.81 & 0.45 & $<0.01$ \\
\hline RNFL/IBILM-ISOS & $0.39(0.02)$ & $0.37(0.03)$ & 6.9 & 0.01 & 0.09 & $0.35(0.03)$ & 22.0 & $<0.01$ & 0.33 & $0.27(0.04)$ & 13.5 & $<0.01$ & 0.19 & 2.75 & 0.07 & 0.04 \\
\hline RNFL/IBIPL-OBOPL & $2.39(0.2)$ & $2.3(0.34)$ & 1.24 & 0.27 & $<0.01$ & $2.6(0.24)$ & 24.4 & $<0.01$ & 0.36 & $2.12(0.3)$ & 14.2 & $<0.01$ & 0.2 & 4.84 & 0.01 & 0.09 \\
\hline
\end{tabular}

Note: The statistically significant $p$-values $(p<0.05)$ are shown in bold text. 
Table 7 ANOVA results on the cohort of MS non-ON eyes with RNFL thickness (global average) in the range of 96 to $106 \mu \mathrm{m}$.

\begin{tabular}{lccccccc} 
& \multicolumn{2}{c}{ Control } & & \multicolumn{4}{c}{ MS non-ON } \\
\cline { 2 - 2 } \cline { 7 - 8 } Variables & Mean (SD) & & Mean (SD) & $F$ & $p$ & $\omega^{2}$ \\
\hline RNFL $_{T}(\mu \mathrm{m})$ & $101.36(5.42)$ & & $99.15(3.21)$ & 2.74 & 0.1 & 0.03 \\
RNFL/IBILM-ISOS & $0.39(0.02)$ & & $0.37(0.01)$ & 5.26 & $\mathbf{0 . 0 3}$ & 0.08 \\
RNFL/IBILM-OBBM & $0.31(0.02)$ & & $0.3(0.01)$ & 4.8 & $\mathbf{0 . 0 3}$ & 0.08 \\
RNFL/IBIPL-ISOS & $0.94(0.08)$ & & $0.89(0.05)$ & 4.2 & $\mathbf{0 . 0 4}$ & 0.07 \\
\hline
\end{tabular}

Note: The statistically significant $p$-values $(p<0.05)$ are shown in bold text.

\section{Discussion}

The peripapillary RNFL thinning in MS patients with or without a history of $\mathrm{ON}$ has been well documented by clinical studies. However, to our knowledge, other peripapillary retinal layers were not investigated extensively for such probable differences in MS patients. In this study, we have calculated the global average of 15 different retinal layer thicknesses for HC, MS-disability groups (non-ON), and MS-ON subjects. The comparison of different retinal thicknesses (global average) for MS non-ON eyes with respect to $\mathrm{HC}$ shows that in addition to $\mathrm{RNFL}_{T}$, the combination of $\mathrm{RNFL}_{T}$ and $\mathrm{GCL}_{T}$ is different in MS patients. On the other hand, the thicknesses involving the RNFL layer and the $\mathrm{GCL}_{T}$ were different in subjects with a history of ON compared to MS non-ON subjects. Thus, the $\mathrm{RNFL}_{T}$ indeed plays an important role in the findings from $\mathrm{MS}$ and $\mathrm{ON}$ subjects.

Ratios of thicknesses, in contrast, have rarely been used in retinal analysis in MS. In this study, we have reported an extensive analysis of different ratios, and for the first time to our knowledge, these ratios can be used to detect subtle retinal differences in MS subjects with and without a history of ON. As many as 105 different ratios were generated from 15 retinal layer thicknesses (global average). We found several significant ratios involving the RFNL in both the MS non-ON and MS-ON groups. However, there were no significant ratios not involving the RFNL for the MS non-ON subjects compared to the HC subjects, though a few such ratios were found in the MS-ON subjects compared to the MS non-ON subjects. As expected, any ratio without the RNFL and GCL was nonsignificant. Further, we found a ratio not involving the RNFL (OBNFLIBIPL/OBNFL-OBOPL) to be different among the MS-disability groups from the ANOVA. Moreover, when the $\mathrm{RNFL}_{T}$ and the ratios of the MS-disability groups were compared to $\mathrm{HC}$, the ANOVA revealed a very interesting result: even though the $\mathrm{RNFL}_{T}$ is not significantly different, several ratios involving the RNFL layer appeared significantly different in the MSmild group with respect to $\mathrm{HC}$ subjects. This is quite remarkable as such ratios without a difference in $\mathrm{RNFL}_{T}$ may suggest the possibility for detecting early retinal changes in MS. It is to be noted that in the comparison of MS-moderate and MS-severe groups, with $\mathrm{HC}$ subjects, as well as among the three disability groups, all show a difference in $\mathrm{RNFL}_{T}$.

We provided a very stringent test of the ratiometric analysis by generating a group of subjects with MS, who had normal $\mathrm{RNFL}_{T}$ and no history of ON, and compared these ratios with those from the HCs. We reported that the cohort of MS non-ON eyes with an $\mathrm{RNFL}_{T}$ (global average) in the range of 96 to $106 \mu \mathrm{m}$ showed some significant ratios involving the RNFL layer, though the $\mathrm{RNFL}_{T}$ or any of the other thicknesses were not statistically different in that cohort. Therefore, this ratiometric analysis may potentially be more sensitive and useful than $\mathrm{RNFL}_{T}$ alone for detecting early or subtle incremental retinal layer thickness changes in MS.

It is noteworthy to mention that we have presented the thicknesses and ratios as significant for which $p$ values are equal or less than 0.01 (instead of just $p<0.05$ ) in Tables $2-6$, but not in Table 7. These values of $p$ are a compromise between type I (the chance of introducing ineffective treatments) and type II (the chance that effective treatments are not discovered) errors. However, for Table 7 (the ANOVA results on the cohort of MS non-ON eyes with RNFL thickness in the range of 96 to $106 \mu \mathrm{m}$ ), we have used $p<0.05$ as the level of significance (there is no ratio with $p<0.01$.). Additionally, we have included the effect size ( $\omega^{2}$ values) in Tables $2-7$, which are appropriate measures to evaluate the quality of the study and the statistical comparisons. The measure of the magnitude of effect in ANOVA is called effect size, which is important alongside investigating and reporting the results of null hypothesis statistical testing. The effect size can be thought of as the correlation between an effect and the dependent variable. The $\omega^{2}$ values estimate the degree of association in the population and are particularly useful because they are not affected by sample size (i.e., less biased compared to other parameters for effect size). Our reported $\omega^{2}$ values indicate medium $(0.06)$ to large $(0.14)$ effects $^{21}$ whenever there is statistical significance (i.e., $p<0.05$ or better).

We recognize that some limitations of our study include the limited and unequal numbers of subjects/eyes, the untested repeatability of our analysis across larger numbers of HC subjects and MS-disability groups, and the possible effects on this ratiometric analysis in eyes with confounding ophthalmic pathologies. Further, we were not able to segment more than six borders due to the limitation in the open source software, which, however, does not place any limitation on our analysis. Also, as we are exploring the possibilities for the ratiometric analysis in this study, there may be a possible chance for type I error, which is another limitation of our study. We are essentially providing the first investigation into the ratiometric analysis that we and others can follow and build upon in the future. Further studies with larger subject groups and prospective follow-up are needed to further validate the findings of this study. The results presented here, however, support the use of ratiometric analyses of retinal layer thicknesses as a means for identifying potentially more sensitive image-based metrics for early and subtle pathological changes in the retinas of MS patients.

\section{Acknowledgments}

We thank Eric Chaney from the Beckman Institute for Advanced Science and Technology for his assistance with managing our IRB protocol and Darold Spillman from the Beckman Institute for Advanced Science and Technology, and Imaging at Illinois, for providing logistical, operations, and information technology support for this project. We also thank Dr. Heather Moss and the anonymous reviewers for their insightful comments and suggestions for this study. This research was supported in part by a grant from the National Institutes of Health (No. NIBIB R01 EB013723, S.A.B.). Additional information can be found at http://biophotonics.illinois.edu. 


\section{References}

1. D. W. Anderson et al., "Revised estimate of the prevalence of multiple sclerosis in the United States," Ann. Neurol. 31(3), 333-336 (1992).

2. H. T. Akcam et al., "Multiple sclerosis and optic nerve: an analysis of retinal nerve fiber layer thickness and color Doppler imaging parameters," Eye 28(10), 1206-1211 (2014).

3. T. L. Sorensen et al., "Optic neuritis as onset manifestation of multiple sclerosis: a nationwide, long-term survey," Neurology 53, 473-478 (1999).

4. J. B. Fisher et al., "Relation of visual function to retinal nerve fiber layer thickness in multiple sclerosis," Ophthalmology 113(2), 324-332 (2006).

5. D. Huang et al., "Optical coherence tomography," Science 254(5035), 1178-1181 (1991).

6. A. F. Fercher, "Optical coherence tomography," J. Biomed. Opt. 1(2), 157-173 (1996).

7. M. E. J. van Velthoven et al., "Recent developments in optical coherence tomography for imaging the retina," Prog. Retinal Eye Res. 26(1), 57-77 (2007).

8. C. Lamirel, N. J. Newman, and V. Biousse, "Optical coherence tomography (OCT) in optic neuritis and multiple sclerosis," Rev. Neurol. 166(12), 978-986 (2010).

9. S. Saidha et al., "Visual dysfunction in multiple sclerosis correlates better with optical coherence tomography derived estimates of macular ganglion cell layer thickness than peripapillary retinal nerve fiber layer thickness," Mult. Scler. J. 17(12), 1449-1463 (2011).

10. R. Kafieh, H. Rabbani, and S. Kermani, "A review of algorithms for segmentation of optical coherence tomography from retina," J. Med. Signals Sens. 3(1), 45-60 (2013).

11. E. Garcia-Martin et al., "Fourier-domain OCT in multiple sclerosis patients: reproducibility and ability to detect retinal nerve fiber layer atrophy," Invest. Ophthalmol. Visual Sci. 52(7), 4124-4131 (2011).

12. S. A. Trip et al., "Retinal nerve fiber layer axonal loss and visual dysfunction in optic neuritis," Ann. Neurol. 58(3), 383-391 (2005).

13. F. Costello, "Evaluating the use of optical coherence tomography in optic neuritis," Mult. Scler. Int. 2011, 148394 (2011).

14. S. Saidha et al., "Primary retinal pathology in multiple sclerosis as detected by optical coherence tomography," Brain 134, 518-533 (2011).

15. Y. Kita et al., "Ability of optical coherence tomography-determined ganglion cell complex thickness to total retinal thickness ratio to diagnose glaucoma," J. Glaucoma 22(9), 757-762 (2013).

16. S. Miri et al., "The avascular zone and neuronal remodeling of the fovea in Parkinson disease," Ann. Clin. Transl. Neurol. 2(2), 196-201 (2015).

17. C. H. Polman et al., "Diagnostic criteria for multiple sclerosis: 2010 revisions to the McDonald criteria," Ann. Neurol. 69(2), 292-302 (2011).

18. J. F. Kurtzke, "Rating neurologic impairment in multiple sclerosis: an expanded disability status scale (EDSS)," Neurology 33(11), 1444-1452 (1983).

19. S. Schippling et al., "Quality control for retinal OCT in multiple sclerosis: validation of the OSCAR-IB criteria," Mult. Scler. J. 21(2), 163-170 (2015).

20. M. A. Mayer et al., "Retinal nerve fiber layer segmentation on FD-OCT scans of normal subjects and glaucoma patients," Biomed. Opt. Express 1(5), 1358-1383 (2010).
21. A. Field, Discovering Statistics Using IBM SPSS Statistics, 4th ed., p. 474, Sage, London (2013).

Basanta Bhaduri received his $\mathrm{PhD}$ in physics from the Indian Institute of Technology, Madras, in 2007. He is currently an assistant professor at the Indian School of Mines, Dhanbad. He was a postdoctoral research fellow at the University of Illinois, Urbana Champaign from 2011 to 2015. He has published more than 40 papers in international journals. His research interests include optical instrumentation, microscopy, optical coherence tomography, and holography. He is a member of SPIE.

Ryan M. Nolan received his BS in bioengineering from University of Pittsburgh and MEng in biomedical engineering from Cornell University. He has extensive experience collaborating with physicians and hospitals developing innovative imaging technologies for integration into clinical environments. He is currently working to commercialize diagnostic imaging technology developed in Prof. Boppart's academic research lab alongside Ryan Shelton in cofounded startup company, PhotoniCare.

Ryan L. Shelton received his BS degree in electrical engineering from Oklahoma State University and his $\mathrm{PhD}$ in biomedical engineering from Texas A\&M University. He has been developing medical imaging technologies for the past 10 years in clinical applications ranging across otology, ophthalmology, and dermatology. Currently, he leads PhotoniCare, a medical device firm developing diagnostic imaging technology for middle ear disease.

Lara A. Pilutti received her doctoral degree in kinesiology from McMaster University, Hamilton, Canada. She is an assistant professor and director of the Clinical Exercise Physiology Laboratory in the Department of Kinesiology and Community Health at the University of Illinois, Urbana-Champaign. Her research focuses on the role of exercise in the management and treatment of disability arising from neurological disorders.

Robert W. Motl is a professor of Kinesiology and Community Health, affiliated to the Division of Neuroscience and director of the Exercise Neuroscience Laboratory at the University of Illinois, UrbanaChampaign. His research interests are in the areas of exercise psychology, measurement, and neuroscience for understanding physical activity and exercise in persons with multiple sclerosis and other neurological conditions.

Stephen A. Boppart is an Abel Bliss professor of engineering in the Departments of Electrical and Computer Engineering, Bioengineering, and Medicine and head of the Biophotonics Imaging Laboratory at the Beckman Institute for Advanced Science and Technology. His research interests include the development of optical imaging technologies for medical applications, with emphasis on translating these to clinical applications. He is a fellow of SPIE, IEEE, AAAS, OSA, and AIMBE. 